\title{
Our love-hate relationship with succinylcholine: Is sugammadex any better?
}

\author{
Orlando Hung, MD $\cdot$ Dolores McKeen, MD $\cdot$ Johannes Huitink, MD, PhD
}

Received: 17 March 2016/Revised: 30 March 2016/Accepted: 20 April 2016/Published online: 3 May 2016

(c) Canadian Anesthesiologists' Society 2016

Some years ago, a colleague provided anesthesia care to a healthy young adult male for an emergency open appendectomy. Standard of care at that time was a rapidsequence induction and intubation (RSI). Following denitrogenation, general anesthesia and muscle relaxation were achieved with fentanyl, thiopental, and succinylcholine. While maintaining cricoid pressure, tracheal intubation was successfully achieved using direct laryngoscopy. The appendectomy proceeded uneventfully. As the surgeon was closing the abdominal wound, he requested additional muscle relaxation, and accordingly, a small dose of intravenous succinylcholine $(20 \mathrm{mg})$ was administered. Within one minute, asystole was witnessed on the electrocardiogram monitor and no carotid pulse was detected. Help was summoned and chest compressions were initiated. Cardiac rhythm, carotid pulse, and oxygen saturation quickly returned to normal after the administration of atropine. At the conclusion of the surgery and after tracheal extubation, the patient was taken to the recovery room awake in stable condition. The patient was informed that cardiac arrest had occurred in the operating room. Despite no apparent long-term postoperative sequelae, medicolegal action was pursued. Many meetings with the patient and his legal team subsequently followed, and although the case did not proceed to trial, all involved were left with a bad memory of the ugly side effects of succinylcholine.

O. Hung, MD ( $\square) \cdot$ D. McKeen, MD

Department of Anesthesia, Pain Management and Perioperative Medicine, Queen Elizabeth II Health Sciences Centre, Dalhousie University, 1278 South Park St, Halifax, NS B3H 2Y9, Canada e-mail: hung192@gmail.com

J. Huitink, MD, PhD

VU University Medical Center, Amsterdam, Netherlands
For decades, we have loved to hate succinylcholine. While most of us love the rapid onset and brief duration of action of succinylcholine at critical moments in airway management, such as RSI, we hate the array of adverse effects it produces-including its potential for cardiac arrest with repeat administration.

Hunt and Taveau discovered suxamethonium, the international non-proprietary name for succinylcholine, in $1906^{1}$, but it was not introduced into clinical practice until the early 1950s. ${ }^{2,3}$ Because of its unique characteristics of rapid onset and short duration of muscle paralysis, succinylcholine enjoyed superiority over other muscle relaxants as the "gold standard" 4 for RSI by clinicians for more than half a century. ${ }^{5}$ According to a survey conducted in several European countries and the United States, ${ }^{6}$ the most desirable features of succinylcholine reported were rapid onset (88\%), short duration (64\%), and effective relaxation (61\%). The thousands of succinylcholine-related papers published since the 1950s have generated an expansive body of literature, including discoveries extending beyond its immediate effects of muscle relaxation. Indeed, it was research into the genetics of inactivation of succinylcholine by plasma cholinesterase that led to the development of the field of pharmacogenetics in the 1950s. ${ }^{7}$ By 1957 , succinylcholine had its own subject heading in Index Medicus! ${ }^{8}$

Unfortunately, the depolarizing nature of succinylcholine is associated with many serious adverse effects of varying clinical significance, and it is therefore contraindicated in many medical conditions, including hyperkalemia, burns, muscular dystrophy, and malignant hyperthermia. In 1992, the United States (US) Food and Drug Administration (FDA) even issued a warning in the Scoline ${ }^{\circledR}$ (suxamethonium chloride; GlaxoSmithKline, USA) package insert after receiving reports of 
intractable cardiac arrests. ${ }^{9}$ All these problems led to decades of succinylcholine research focusing primarily on understanding its serious side effects.

In the decades that followed the discovery of succinylcholine, its shortcomings led researchers to make many attempts to replace the drug with alternate nondepolarizing muscle relaxants with similarly rapid onset and short duration. ${ }^{10}$ Sadly, many promising molecules, including rapacurium, failed to demonstrate acceptable safety profiles. ${ }^{11,12}$ Fortunately, large doses of rocuronium (e.g., $1.2 \mathrm{mg} \cdot \mathrm{kg}^{-1}$ ) have been shown to have comparable onset times to succinylcholine in the setting of RSI. $^{5}$ The downside is that many clinicians are reluctant to administer such large doses due to the associated prolonged paralysis and apnea, particularly in patients whose lungs may be difficult to ventilate and oxygenate. So, the search continues for a replacement for succinylcholine.

In 2007, Dr. Anton Bom serendipitously discovered sugammadex, a modified gamma ( $\left(\right.$ )-cyclodextrin, ${ }^{13}$ while he was searching for a new solvent for rocuronium bromide. ${ }^{14}$ It is the first selective relaxant-binding agent that encapsulates and binds neuromuscular blocking agents (NMBAs) by exerting a chelating action and effectively terminating muscle paralysis of an NMBA. Sugammadex has a negatively charged lipophilic core and a hydrophilic periphery that has strong affinity for amino steroid NMBAs, forming tight complexes in a 1:1 ratio with rocuronium. In contrast to traditional cholinesterase inhibitor reversal therapy, inactivation of rocuronium by sugammadex encapsulation has been shown to be fast, effective, and relatively safe with few reported side effects. The European Union approved the use of sugammadex in 2008, followed by Australia, New Zealand, and Venezuela in 2009, Japan and other parts of Asia in 2010, and more recently, the United States (2015) and Canada (2016). A recent report indicated that there have been approximately 11.5 million clinical sugammadex exposures since its introduction. ${ }^{15}$ This is exciting news for the anesthesia community, since we now have a drug that can quickly reverse the effect of high-dose rocuronium required for RSI-it potentially allows us to stop using succinylcholine. ${ }^{16,17}$

Following the introduction of sugammadex, we recently sought to determine its impact on the clinical use of succinylcholine by informally soliciting information from colleagues in ten countries around the globe (Australia, Germany, Italy, Malaysia, New Zealand, Saudi Arabia, Singapore, Switzerland, United Kingdom, and Venezuela) and focusing solely on the issue of RSI. Four questions were asked: 1) Do you still employ RSI? 2) Do you use succinylcholine for RSI? 3) Have you used succinylcholine during the last 12 months? 4) When was the last time you used succinylcholine? While all respondents performed
RSI, only $70 \%$ of respondents used succinylcholine for RSI. Many respondents claimed that they rarely use succinylcholine, and 50\% could not remember the last time they used it. Interestingly, some had not used succinylcholine for more than ten years, well before sugammadex was introduced. A recent national survey in the United Kingdom reported similar findings regarding the use of succinylcholine for RSI. ${ }^{18}$ Most respondents (56\%) used succinylcholine for RSI, but they used rocuronium in selected patients. Similarly, $20 \%$ of the respondents usually used rocuronium for RSI, but they chose succinylcholine for some selected patients. The investigators concluded that "...introduction of sugammadex might have contributed to the reduced use of suxamethonium..."

Recognizing that the recent change in the usage pattern of succinylcholine is likely multifactorial and context sensitive, it may well be that sugammadex finally brings our ambivalent relationship with succinylcholine to an end. Sugammadex has been a "game changer" for airway management in many countries. There has been a drop in the use of atracurium, mivacurium, and succinylcholine in recent years, with many clinicians now using rocuronium for muscle relaxation along with sugammadex as a rescue for failed intubation. Furthermore, many clinicians use sugammadex to shorten the recovery time for short procedures requiring deep muscular block (e.g., head and neck surgeries or laparoscopic procedures).

Nevertheless, abandoning the use of succinylcholine is not that simple, and reaching a consensus may take time. Many still love succinylcholine because it is considered the best drug available for excellent intubation conditions in RSI. While many reports indicate that, with appropriate dosing, succinylcholine and rocuronium have similar clinically acceptable intubation conditions and onset time, succinylcholine consistently produces better intubation conditions with higher satisfaction scores. ${ }^{5}$ Furthermore, its low cost and ubiquitous nature make it an easy choice to reach for in the operating room. But we still hate it for its adverse effects.

Some also hate that succinylcholine "got lucky", pointing to its introduction in an era when regulatory scrutiny was less stringent. Its profile of serious side effects compared with alternatives would likely never survive the oversight of contemporary research ethics boards or the regulatory scrutiny of the US FDA and the Health Protection Branch (HPB) of Health Canada. Under the present rigorous regulatory evaluation process, the path from discovery to clinical use has been difficult for many non-depolarizing NMBAs and sugammadex. At the same time that sugammadex obtained European Union regulatory approval, it failed to receive FDA approvaleven though an FDA advisory committee unanimously recommended approval—citing concerns related to 
hypersensitivity and anaphylactic reactions. ${ }^{15}$ Eight years later and after many additional clinical trials, sugammadex finally received FDA (December 2015) and HPB (February 2016) approval.

Though sugammadex is not without problems, ${ }^{19,20}$ data from phase 1-3 clinical trials as well as post-marketing data from millions of patients have shown that sugammadex is safe. There is only a rare risk $(<1 \%)$ of anaphylactic reactions with sugammadex, and these are treatable with standard therapy. ${ }^{15,21}$ With the proven efficacy and safety profile of sugammadex (in combination with high-dose rocuronium), clinicians around the world have not yet embraced it as a replacement for the riskier succinylcholine. The slow rate of acceptance of sugammadex is no doubt partly due to its cost.

Like many historically important drugs, succinylcholine will likely eventually fade into obscurity, not solely because of the availability of sugammadex, but rather by continued NMBA development, improved failed airway exit strategies, and perhaps eventual reduction in sugammadex drug costs. Sugammadex may play an important role in RSI for reversal of high-dose rocuronium in those rare situations when spontaneous ventilation must be restored after failed intubation and oxygenation. There are other potential uses of sugammadex; for example, it will likely play a major role in the management of patients whose operation is completed much earlier than anticipated or when residual muscle paralysis exists at the conclusion of surgery.

During the last several decades, clinicians have been striving to improve not only the outcomes of RSI (by using drugs with better safety profiles) but also the capacity to provide rapid reversal of paralysis should RSI fail. In the meantime, however, strategic planning for the actual airway management in RSI has been largely ignored. The keys to improve outcomes include a thorough assessment of the airway and planned strategies prior to the intervention so as to manage unanticipated difficulties encountered during RSI. ${ }^{22,23}$ Although the immediate reversal of rocuronium and consequent return of muscle function is a frequently raised potential scenario for the use of sugammadex, there is currently a lack of convincing evidence to suggest that this approach has been successful in providing oxygenation and ventilation in the face of a "cannot intubate, cannot oxygenate" failed airway. In this situation and in the presence of hypoxemia, the standard of care for a failed RSI requires the clinician to resort to a front-of-neck technique for immediate access while attempting to reverse the effect of rocuronium using sugammadex. ${ }^{23-25}$

It appears that we may also have a love-hate relationship with sugammadex. We seem ready to love sugammadex even though it has some clinical limitations, but we also hate its cost. And so, it appears that we aren't yet ready to abandon succinylcholine. It will no doubt remain on the hospital formulary, but its clinical use will likely decrease over time. Like all ambivalent relationships that we closely examine, we often come to the sad conclusion that we deserve better.

\section{Succinylcholine, nous t'aimons, nous te haïssons: et toi, suggammadex, es-tu mieux loti?}

Il y a de cela plusieurs années, un collègue a prodigué des soins anesthésiques à un jeune homme en bonne santé pour une appendicectomie ouverte urgente. À l'époque, l'induction et l'intubation en séquence rapide (ISR) constituaient la norme de soins. À la suite de la préoxygénation, l'anesthésiologiste a utilisé un mélange de fentanyl, thiopental et succinylcholine pour réaliser l'anesthésie générale et la relaxation musculaire. Tout en maintenant la pression cricoïdienne, l'intubation trachéale a été réalisée par laryngoscopie directe, et l'appendicectomie s'est déroulée sans incident. Alors que le chirurgien suturait la plaie abdominale, il a demandé une relaxation musculaire supplémentaire, et une petite dose de succinylcholine intraveineuse $(20 \mathrm{mg})$ a donc été administrée. Dans la minute qui a suivi, une asystolie est apparue sur le moniteur de l'électrocardiogramme et le pouls carotidien n'était plus détectable. Une assistance a été requise et le massage cardiaque amorcé. Le rythme cardiaque, le pouls carotidien et la saturation en oxygène se sont rapidement rétablis après l'administration d'atropine. Après la chirurgie et l'extubation trachéale, le patient était éveillé et stable quand il a été transféré en salle de réveil. Le patient a été informé qu'il avait subi un arrêt cardiaque en salle d'opération. Malgré l'absence de séquelles postopératoires apparentes à long terme, une poursuite médicolégale a été intentée. De nombreuses rencontres se sont ensuivies avec le patient et son équipe légale et, bien que la cause ne soit pas passée devant les tribunaux, toutes les parties impliquées gardent un très mauvais souvenir des affreux effets secondaires de la succinylcholine.

Durant des dizaines d'années, nous avons adoré haïr la succinylcholine. Bien que la plupart d'entre nous chérissent sa rapidité d'action et sa brève durée d'action dans les moments cruciaux de la prise en charge des voies aériennes, tels que pendant l'ISR, nous haïssons la pléiade d'effets secondaires néfastes qu'elle entraîne - y compris l'arrêt cardiaque potentiel dû à des administrations répétées. 
Hunt et Taveau ont découvert le suxaméthonium, le nom non breveté international de la succinylcholine, en $190^{1,6}$ toutefois, ce curare n'a été introduit dans la pratique clinique qu'au début des années $1950 .^{2,3}$ En raison de ses propriétés uniques, soit un court délai d'action et une curarisation de courte durée, la succinylcholine a été préférée aux autres curares et considérée "l'étalon or » pour l'ISR par les cliniciens pendant plus d'un demi-siècle. ${ }^{5}$ Selon un sondage réalisé dans plusieurs pays européens et aux États-Unis, ${ }^{6}$ les caractéristiques les plus appréciées de la succinylcholine sont son délai d'action rapide ( $88 \%)$, sa courte durée d'action $(64 \%)$, et l'efficacité de la relaxation obtenue (61\%). Les milliers d'articles portant sur la succinylcholine publiés depuis les années 1950 ont généré un corpus de littérature impressionnant, qui comprend des observations qui vont bien au-delà de ses effets immédiats de curarisation. En effet, c'est grâce aux recherches sur la génétique de l'inactivation de la succinylcholine par la cholinestérase plasmatique que le développement du domaine de la pharmacogénétique a pris son essor dans les années $1950{ }^{7}$ En 1957, la succinylcholine possédait même sa propre vedette-matière dans l'Index Medicus! $!^{8}$

Malheureusement, la nature dépolarisante de la succinylcholine est associée à de nombreux effets secondaires néfastes dont l'importance clinique varie. C'est pour cette raison qu'elle est contre-indiquée dans de nombreuses conditions médicales, notamment en cas d'hyperkaliémie, de brûlures, de dystrophie musculaire et d'hyperthermie maligne. En 1992, la Food and Drug Administration (FDA) américaine a même fait figurer un avertissement dans l'insert du paquet de Scoline ${ }^{\circledR}$ (chlorure de suxaméthonium; GlaxoSmithKline, États-Unis) après avoir reçu des comptes rendus d'arrêts cardiaques réfractaires. ${ }^{9}$ Tous ces problèmes ont mené à des dizaines d'années de recherche sur la succinylcholine, dont l'objectif principal était de comprendre ses effets secondaires graves.

Dans les décennies suivant la découverte de la succinylcholine, ses défauts ont mené les chercheurs à tenter, à plusieurs reprises, de remplacer cet agent par d'autres curares non dépolarisants présentant également un délai d'action rapide et une courte durée d'action. ${ }^{10}$ Malheureusement, les profils d'innocuité de plusieurs molécules prometteuses, y compris le rapacurium, se sont avérés inacceptables. ${ }^{11,12}$ Heureusement, il a été démontré que de fortes doses de rocuronium (par ex. $1,2 \mathrm{mg} \cdot \mathrm{kg}^{-1}$ ) présentaient des délais d'action comparables à la succinylcholine en cas d'ISR. ${ }^{5}$ L'inconvénient, c'est que bon nombre de cliniciens rechignent à administrer des doses aussi importantes en raison de la paralysie et de l'apnée prolongées qui y sont associées, particulièrement chez les patients dont les poumons pourraient être difficiles à ventiler et à oxygéner. La quête pour un remplaçant de la succinylcholine se poursuit toujours.

En 2007, le Dr Anton Bom a, par un heureux hasard, découvert le sugammadex, une gamma $(\Upsilon)$-cyclodextrine modifiée, ${ }^{13}$ alors qu'il cherchait un nouveau solvant pour le bromure de rocuronium. ${ }^{14}$ Il s'agit du premier agent se liant sélectivement à un curare qui encapsule et lie les bloqueurs neuromusculaires en exerçant une action chélatrice et en interrompant par conséquent la paralysie musculaire d'un curare. Le sugammadex a un cœur lipophile chargé négativement et une couronne hydrophile qui possède une forte affinité pour les curares aminostéroïdes, formant des complexes serrés dans un rapport 1:1 avec le rocuronium. Comparativement au traitement conventionnel de renversement par inhibition de la cholinestérase, il a été démontré que l'inactivation du rocuronium par encapsulation au sugammadex était rapide, efficace et relativement sécuritaire, avec peu d'effets secondaires rapportés. L'utilisation du sugammadex a été approuvée par l'Union européenne en 2008, suivie par l'Australie, la Nouvelle-Zélande et le Venezuela en 2009, puis le Japon et d'autres parties de l'Asie en 2010 et, plus récemment, les États-Unis (2015) et le Canada (2016). Un compte rendu récent a indiqué qu'il y a eu environ 11,5 millions d'expositions cliniques au sugammadex depuis son introduction. ${ }^{15}$ Ces nouvelles sont réjouissantes pour la communauté de l'anesthésie, étant donné que nous disposons désormais d'un médicament qui peut rapidement neutraliser l'effet des fortes doses de rocuronium nécessaires à l'ISR - et il nous permettra peut-être d'arrêter d'utiliser la succinylcholine. ${ }^{16,17}$

Suite à l'introduction du sugammadex, nous avons récemment tenté de déterminer son impact sur l'utilisation clinique de la succinylcholine en demandant de l'information, de façon informelle, à nos collègues de dix pays dans le monde entier (Australie, Allemagne, Italie, Malaisie, Nouvelle-Zélande, Arabie saoudite, Singapour, Suisse, Royaume-Uni et Venezuela) et en nous concentrant exclusivement sur la question de l'ISR. Nous leur avons posé quatre questions : 1) Employez-vous toujours l'ISR? 2) Utilisez-vous la succinylcholine pour l'ISR? 3) Avez-vous utilisé la succinylcholine au cours des 12 derniers mois? 4) Quand avez-vous utilisé la succinylcholine pour la dernière fois? Alors que tous les répondants pratiquaient encore l'ISR, seuls $70 \%$ utilisaient la succinylcholine pour l'ISR. Bon nombre de répondants ont déclaré n'utiliser que rarement la succinylcholine, et 50 $\%$ ne se rappelaient plus de la dernière fois qu'ils l'avaient utilisée. Fait intéressant, certains n'utilisaient plus la succinylcholine depuis plus de dix ans - soit bien avant l'introduction du sugammadex. Un sondage récent réalisé dans l'ensemble du Royaume-Uni a rapporté des résultats 
semblables quant à l'utilisation de succinylcholine pour l'ISR. ${ }^{18}$ La plupart des répondants $(56 \%)$ utilisaient la succinylcholine pour l'ISR, mais le rocuronium chez certains patients. De la même façon, $20 \%$ des répondants utilisaient en général le rocuronium pour l'ISR, mais ont choisi la succinylcholine pour certains patients. Les chercheurs ont conclu que « ...l'introduction du sugammadex pourrait avoir contribué à la réduction de l'utilisation de suxaméthonium... »

Reconnaissant que les changements récents dans les habitudes d'utilisation de la succinylcholine sont probablement plurifactoriels et liés à certains contextes, il se pourrait bien que le sugammadex signe enfin l'arrêt de mort de notre relation ambivalente avec la succinylcholine. Dans de nombreux pays, le sugammadex a complètement révolutionné la prise en charge des voies aériennes. L'utilisation de l'atracurium, du mivacurium et de la succinylcholine a chuté ces dernières années; en effet, plusieurs cliniciens utilisent désormais le rocuronium comme curare, avec le sugammadex comme agent de sauvetage en cas d'échec de l'intubation. De plus, bon nombre de cliniciens ont recours au sugammadex pour raccourcir le délai de récupération lors d'interventions de courte durée nécessitant un bloc musculaire profond (par ex., chirurgies de la tête et du cou ou interventions par laparoscopie).

Toutefois, l'abandon de la succinylcholine n'est pas si simple, et le consensus pourrait prendre du temps à d'être atteint. Bon nombre de cliniciens aiment toujours la succinylcholine parce qu'elle est considérée comme le meilleur agent disponible pour procurer d'excellentes conditions d'intubation en cas d'ISR. Bien que plusieurs comptes rendus indiquent que, lorsque bien dosés, la succinylcholine et le rocuronium procurent tous deux des conditions d'intubation et un délai d'action acceptables d'un point de vue clinique, la succinylcholine procure toujours de meilleures conditions d'intubation avec des scores de satisfaction plus élevés. ${ }^{5}$ En outre, son faible coût et son omniprésence la rendent facile d'accès dans la salle d'opération. Néanmoins, nous la détestons tout de même en raison de ses effets secondaires.

Certains détestent également le fait que la succinylcholine "a été chanceuse », en faisant référence à son introduction, qui a eu lieu à un moment où les évaluations réglementaires étaient moins strictes. En raison de son profil d'effets secondaires graves, comparativement à ses alternatives, la succinylcholine ne recevrait probablement pas l'approbation des comités d'éthique de la recherche actuels, et elle ne passerait pas l'examen réglementaire de la FDA américaine ni de la Direction générale de la protection de la santé (DGPS) de Santé Canada. En raison du rigoureux processus d'évaluation réglementaire actuel, le cheminement entre la découverte et l'utilisation clinique a été difficile pour bon nombre de bloqueurs neuromusculaires non dépolarisants et pour le sugammadex. Alors que le sugammadex recevait l'approbation réglementaire de l'Union européenne, il ne la recevait pas de la FDA, en raison d'inquiétudes liées à une hypersensibilité et à des réactions anaphylactiques et ce, même si un comité de consultation de la FDA avait unanimement recommandé son approbation. ${ }^{15}$ Huit ans et plusieurs autres études cliniques plus tard, le sugammadex a enfin été approuvé par la FDA (décembre 2015) et la DGPS (février 2016).

Bien que le sugammadex ne soit pas parfait, ${ }^{19,20}$ les données d'études cliniques de phase 1-3 ainsi que les données recueillies depuis sa commercialisation chez des millions de patients démontrent que cet agent est sécuritaire. Il n'existe qu'un risque rare $(<1 \%)$ de réactions anaphylactiques avec le sugammadex, et ces réactions sont traitables avec la thérapie usuelle. ${ }^{15,21}$ Malgré l'efficacité prouvée et le profil d'innocuité du sugammadex (lorsqu'il est combiné à une forte dose de rocuronium), les cliniciens de par le monde ne l'ont pas encore adopté pour remplacer la succinylcholine, pourtant bien plus risquée. La lenteur de son adoption est certainement due en partie à son coût.

Tout comme c'est le cas pour plusieurs médicaments d'importance historique, il est probable que la succinylcholine sera finalement oubliée, non seulement à cause de la disponibilité du sugammadex, mais aussi grâce à la mise au point incessante de nouveaux bloqueurs neuromusculaires, de stratégies améliorées pour sauvegarder des voies aériennes compromises et peut-être à la réduction éventuelle des coûts du sugammadex. Le sugammadex pourrait jouer un rôle important dans l'ISR pour neutraliser de fortes doses de rocuronium dans les rares situations où il faut rétablir la ventilation spontanée après un échec d'intubation et d'oxygénation. Il existe d'autres utilisations potentielles du sugammadex : par exemple, cet agent jouera probablement un rôle majeur dans la prise en charge des patients dont l'opération se termine beaucoup plus tôt que prévu ou lorsqu'il demeure une paralysie musculaire résiduelle à la fin de la chirurgie.

$\mathrm{Au}$ cours des dernières décennies, les cliniciens se sont efforcés d'améliorer non seulement les pronostics après une ISR (en utilisant des médicaments avec de meilleurs profils d'innocuité) mais aussi la capacité à neutraliser rapidement la paralysie en cas d'échec de l'ISR. En attendant, toutefois, la planification stratégique pour la véritable prise en charge des voies aériennes en cas d'ISR a été en grande partie ignorée. Parmi les clés à l'amélioration des pronostics, citons une évaluation exhaustive des voies aériennes et des stratégies planifiées avant l'intervention de façon à savoir prendre en charge les difficultés inattendues rencontrées pendant l'ISR. ${ }^{22,23}$ Bien que la neutralisation immédiate du rocuronium et le retour consécutif de la 
fonction musculaire constituent une utilisation potentielle du sugammadex fréquemment suggérée, nous manquons pour le moment de données probantes pour nous convaincre que cette approche peut procurer une oxygénation et une ventilation dans un scénario de voies aériennes « impossible d'intuber, impossible d'oxygéner ». Dans une telle situation et en présence d'hypoxémie, la norme de soins pour un échec d'ISR requiert du clinicien qu'il utilise une technique à l'avant du cou pour obtenir un accès immédiat tout en essayant de neutraliser l'effet du rocuronium à l'aide de sugammadex. ${ }^{23-25}$

Il semblerait donc que nous pourrions également développer une relation d'amour-haine avec le sugammadex. Nous semblons prêts à l'adorer malgré ses limites cliniques - tout en haïssant son coût. Nous ne sommes probablement pas tout à fait prêts à abandonner la succinylcholine. Cet agent va indubitablement demeurer sur les formulaires d'hôpitaux, mais son utilisation clinique va probablement diminuer au fil du temps. Comme c'est le cas dans toutes les relations ambivalentes, si nous les examinons de près, nous en venons souvent à la triste conclusion que nous méritons mieux.

\section{Financial support None.}

Conflict of interest Dr. Dolores McKeen has previously received consulting honoraria and conducted research funded by Merck Canada.

Editorial responsibility This submission was handled by Dr. Hilary P. Grocott, Editor-in-Chief, Canadian Journal of Anesthesia.

\section{Soutien financier Aucun.}

Conflit d'intérêt Par le passé, la Dre Dolores McKeen a reçu des honoraires de consultante et réalisé des travaux de recherche financés par Merck Canada.

Responsabilité éditoriale Cet article a été traité par Dr Hilary P. Grocott, rédacteur en chef, Journal canadien d'anesthésie.

\section{References}

1. Hunt $R$, Taveau $R$. On the physiologic actions of certain cholin derivatives and new methods for detecting cholin. Br Med J 1906; 2: 1788-91.

2. Bovet D. Some aspects of the relationship between chemical constitution and curare-like activity. Ann N Y Acad Sci 1951; 54: 407-37.

3. Martyn J, Durieux ME. Succinylcholine: new insights into mechanisms of action of an old drug. Anesthesiology 2006; 104: 633-4.

4. Miller R. Will succinylcholine ever disappear? Anesth Analg 2004; 98: 1674-5.
5. Tran DT, Newton EK, Mount VA, Lee JS, Wells GA, Perry JJ. Rocuronium versus succinylcholine for rapid sequence induction intubation. Cochrane Database Syst Rev 2015; 10: CD002788.

6. Karanovic N, Carev M, Kardum G, et al. Succinylcholine use in adult anesthesia - A multinational questionnaire survey. Coll Antropol 2011; 35(Suppl 1): 183-90.

7. Kalow $W$. Human pharmacogenomics: the development of a science. Hum Genomics 2004; 1: 375-80.

8. Dorkins HR. Suxamethonium-the development of a modern drug from 1906 to the present day. Med Hist 1982; 26: 145-68.

9. Rosenberg H, Gronert GA. Intractable cardiac arrest in children given succinylcholine. Anesthesiology 1992; 77: 1054.

10. Caldwell JE. The continuing search for a succinylcholine replacement. Anesthesiology 2004; 100: 763-4.

11. Belmont MR, Lien CA, Tjan J, et al. Clinical pharmacology of GW280430A in humans. Anesthesiology 2004; 100: 768-73.

12. Sparr HJ, Beaufort TM, Fuchs-Buder T. Newer neuromuscular blocking agents: how do they compare with established agents? Drugs 2001; 61: 919-42.

13. Naguib $M$. Sugammadex: another milestone in clinical neuromuscular pharmacology. Anesth Analg 2007; 104: 575-81.

14. Booij LH. Cyclodextrins and the emergence of sugammadex. Anaesthesia 2009; 64(Suppl 1): 31-7.

15. Murphy G. The development and regulatory history of sugammadex in the United States. APSF Newsletter 2016; 30: 45-76.

16. Caldwell JE, Miller RD. Clinical implications of sugammadex. Anaesthesia 2009; 64(Suppl 1): 66-72.

17. Lee C. Goodbye suxamethonium! Anaesthesia 2009; 64(Suppl 1): 73-81.

18. Sajayan A, Wicker J, Ungureanu N, Mendonca C, Kimani PK. Current practice of rapid sequence induction of anaesthesia in the UK - a national survey. Br J Anaesth 2016. DOI:10.1093/bja/ aew017.

19. Bisschops MM, Holleman C, Huitink JM. Can sugammadex save a patient in a simulated 'cannot intubate, cannot ventilate' situation? Anaesthesia 2010; 65: 936-41.

20. Ikeda-Miyagawa $Y$, Kihara $T$, Matsuda $R$. Case of negative pressure pulmonary edema after administration of sugammadex under general anesthesia with laryngeal mask airway (Japanese). Masui 2014; 63: 1362-5.

21. FDA Advisory Committee. Sugammadex Injection. NDA 22225: Advisory Committee Briefing Materials. Merck Sharp \& Dohme Corporation; 2015. Available from URL: http://www.fda.gov/ downloads/AdvisoryCommittees/CommitteesMeetingMaterials/ Drugs/AnestheticAndAnalgesicDrugProductsAdvisoryCommittee/ UCM470718.pdf (accessed March 2016).

22. Law JA, Broemling N, Cooper RM, et al. The difficult airway with recommendations for management-part 2-the anticipated difficult airway. Can J Anesth 2013; 60: 1119-38.

23. Law JA, Broemling $N$, Cooper RM, et al. The difficult airway with recommendations for management-part 1-difficult tracheal intubation encountered in an unconscious/induced patient. Can J Anesth 2013; 60: 1089-118.

24. Frerk C, Mitchell VS, McNarry AF, et al. Difficult Airway Society 2015 guidelines for management of unanticipated difficult intubation in adults. Br J Anaesth 2015; 115: 827-48.

25. Brindley $P G$, Beed $M$, Duggan LV, Hung O, Murphy $M F$. Updating our approach to the difficult and failed airway: time to “stop and think". Can J Anesth 2016; 63: 373-81. 